\title{
马来酰亚胺氮氧自由基标记细胞膜和 活细胞的电子自旋共振波谱的研究
}

\author{
析文娟 张清刚 张建中* 李益新 范景玮 \\ (中国科学院生物物研究所)
}

近年来由于自旋标记技术的引人，大大地扩大了电子自旋共振（ESR）技术在生物物理 学方面的应用.

自旋标记方法就是把一个理化特性已十分清楚的顺磁体, 例如稳定的有机氮氧自由基棁 到或接到生物大分子上去, 通过有关 ESR 参数的变化可以探测生物大分子的空间构象, 小分 子与生物大分子体系相互作用等, 还可以研究活细胞中的分子结构的变化 ${ }^{[1]}$.

马来酰亚胺氮氧自由基能与具有硫氢 $-\mathrm{SH}$ 基团的生物分子形成特异性结合 ${ }^{[2]}$. 已知 $-\mathrm{SH}$ 基的变化同膜和细胞的各种重要生理现象有关. 例如与细胞分裂、恶性增生有关. 因此应用 马来酰亚胺氮氧自由基和 ESR 相结合的技术, 从亚分子水平来研究细胞膜和活细胞中硫氢 基的变化是有一定意义的 ${ }^{[3,4]}$.

\section{材料与方法}

我们合成了 $\mathrm{N}-(1$-氧基-2,2,6,6-四甲基-4-哌啶)马来酰亚胺氮氧自由基. 经过元素 分析、红外光谱、核磁共振、电子自旋共振等方法的鉴定,证明质量良好 ${ }^{[5]}$.

我们制备了羊红细胞膜. 自旋标记方法如下: 取羊红细胞膜 $0.25 \mathrm{ml}$ 悬浮在 $1 \mathrm{ml}$ 含有 $10^{-3} \mathrm{MHgCl}_{2}$ 的 $0.2 \mathrm{M}$ 磷酸缓冲液 ( $\mathrm{pH7} .4$ ) 中, 在 $5^{\circ} \mathrm{C}$ 静置 22 小时, 然后离心除去上清液, 再用上述缓冲液洗涤五、次,最后将红细胞膜悬浮在含有 $10^{-3} \mathrm{M}$ 马来酰亚胺氮氧自由基的磷酸 缓冲液中, 经 3 小时 $\left(5^{\circ} \mathrm{C}\right)$, 离心除去上清液, 再用磷酸缓冲液洗涤五次, 即可作为被检测的样 品. 同时制备另一组不加 $\mathrm{HgCl}_{2}$ 处理的样品.

实验用细胞为两株中国地鼠卵巢细胞. 一株是在我室培养的第 16-20 代细胞（简称 CHO-I); 另一株是已培养了 80 代以上的转化细胞(简称 $\mathrm{CHO}-\mathrm{II}$ ). 每次传代至含有 $85 \%$ Eagle 和 $15 \%$ 小牛血清的培养液中, 实验使用传代后第 4-5 天贴壁生长的细胞。在其培养液 中加人抗肿瘤药物长春新碱, 药物浓度为 $0.04 \mathrm{mg} / \mathrm{ml}$, 在 $37^{\circ} \mathrm{C}$ 培养 15 小时, 然后倾去培养 液, 加人含有 $10^{-3} M$ 马来酰亚胺氮氧自由基的磷酸缓冲液 (pH7.4), 在 $37^{\circ} \mathrm{C}$ 继续培养 1 小 时, 最后将培养细胞转至离心管中, 离心除去上清液, 用缓冲液洗涤五次, 即可测定. 对照组则 不加抗肿㿔药物。

本文 1980 年 11 月 1 日收到。

* 中国科技大学研究生院。 
ESR 波谱测定,系使用我所四室 404 型高频小调场式电子自旋共振波谱仪, 工作在 X-波 段. 对所有生物样品都在室温进行测定.

\section{结果与讨论}

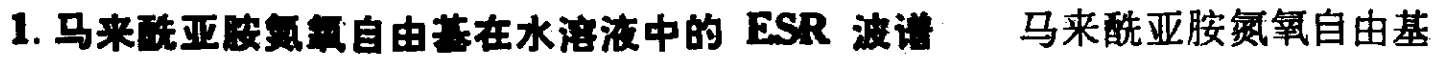<smiles>CC1(C)CC(N2C(=O)C=CC2=O)CC(C)(C)N1O</smiles>

又名顺丁烯二酰亚胺氮氧自由基, 具有高度的稳定性, 这是由于氮原子邻近的两个 $\alpha$-碳原子 上的 $\mathrm{H}$ 各被二个甲基所取代. 不成对电子主要定域在氮原子的 $2 \mathrm{P}_{\mathrm{x}}$ 轨道. 图 1 为在室温测 定的马来酰亚胺氧氧自由基水溶液的 ESR 波谱,呈现出等强度、等距离的三峰谱线. 此波谱 可以用自旋哈密顿 $\hat{e}$ 描述 ${ }^{[6]}$ :

$$
\hat{\mathcal{C}}=\beta \boldsymbol{S} \cdot \boldsymbol{g} \cdot \boldsymbol{H}+\boldsymbol{S} \cdot \boldsymbol{A} \cdot \boldsymbol{I},
$$

其中 $\boldsymbol{S}$ 和 $\boldsymbol{I}$ 分别是电子自旋和核自旋算符, $\boldsymbol{H}$ 为磁场矢量, $\boldsymbol{g}$ 和 $\boldsymbol{A}$ 分别为 $\boldsymbol{g}$ 张量和超精 细偶合张量 (皆为二级张量)， $\boldsymbol{g}$ 表征不成对电子自旋和轨道角动量之间的相互作用; $\boldsymbol{A}$ 表征 不成对电子和 ${ }^{14} \mathrm{~N}$ 核自旋之间的相互作用. 理论和实验均表明, 该自由基的 ESR 波谱表现为 等强度、等间隔的三线谱. 由于 $\boldsymbol{A}$ 和 $\boldsymbol{g}$ 的各向异性, 使得自由基 ESR 谱对其运动性极为敏 感. 在稀释的低粘度介质中, 由于分子的快速翻滚运动使 $\boldsymbol{A}$ 张量的各向异性的部分平均为

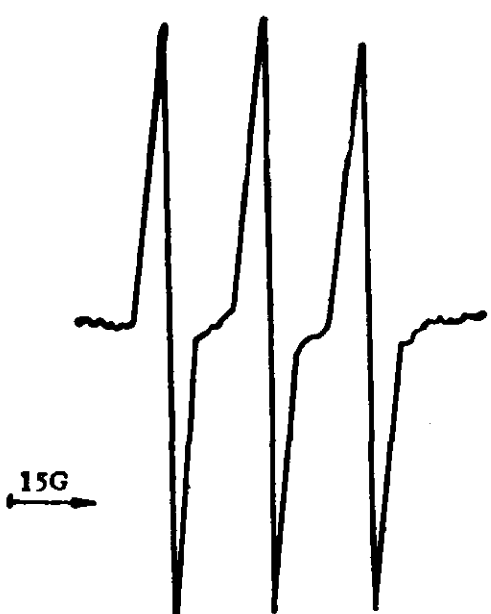

图 1 马来酰亚胺氮篻自由基的 ESR 谱 $10^{-4} M$ 水洨液,室湜

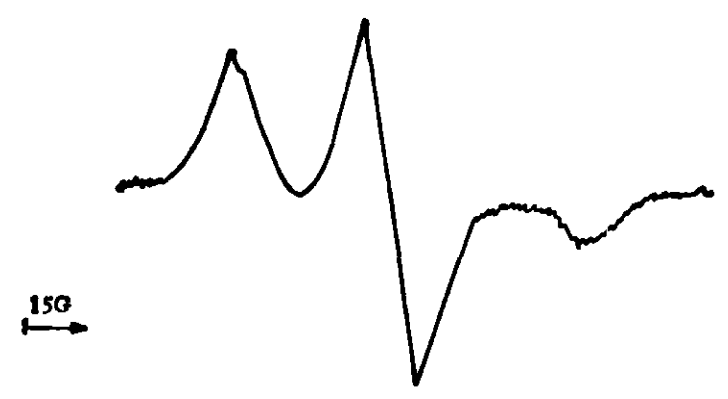

图 2 马来酰亚胺圂篻自由基的 ESR 谱 $10^{-4} M$ 水溶洨, $-196^{\circ} \mathrm{C}$ 
零, 所以图 1 表现出约 15 高斯的各向同性的超精细分裂. 这种与相关时间小于 $10^{-9}$ 秒相对 应的波谱 ${ }^{[1]}$ 称为弱固定化的波谱. 随着粘度的增加, 就逐渐表现出 $\boldsymbol{A}$ 和 $\boldsymbol{g}$ 的各向异性, 三条 等距离的谱线的裂距和线宽也逐渐增加, 高场线变得最快, 低场线次之, 中场线最慢. 图 2 为 马来酰亚胺自旋标记物在 $-196^{\circ} \mathrm{C}$ 时的 ESR 谱, 称为强固定化波谱. 两外缘线之间的距离约 为 60 高斯.

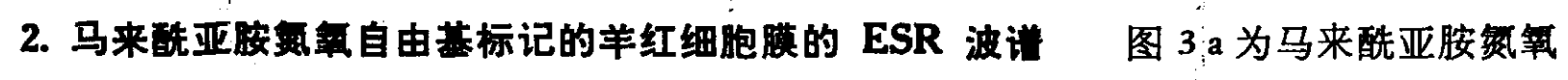
自由基所标记的羊红细胞的 ESR 波谱. 实际上是强固定化和弱固定化两种波谱的叠加. 两种 波谱对应着硫氢基的两种不同位置. 弱固定化表示马来酰亚胺氮氧自由基与处于表面结构的 硫氢基形成特异性的结合, 具有较大的自由度; 强固定化则表示马来酰亚胺氮氧自由基与处于 内部结构的硫氢基形成特异性结合, 其运动受到限制.

图 $3 \mathrm{~b}$ 为羊红细胞膜预先用 $10^{-3} \mathrm{MHgCl}_{2}$ 处理封闭膜上的硫氢基团, 再进行自旋标记所 得的 ESR 谱, 与图 $3 \mathrm{a}$ 相比, 其信号强度显著下降. 从而证明马来酰亚胺氮氧自由基主要结 合到膜上的硫氢基团. 图 $3 \mathrm{c}$ 为对照的未经自旋标记的羊红细胞膜的 ESR 波谱.

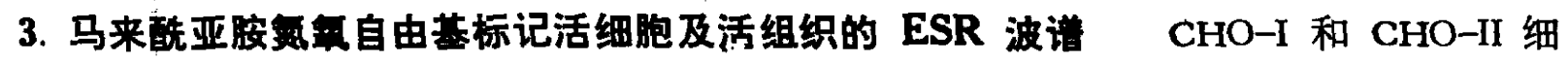
胞在标记前后均经 $2 \%$ 台盼蓝染料染色, 显微镜观察其着色情况和形态, 证明经马来酰亚胺氮 氧自由基标记后的细胞活力与对照组基本相同.

我们用马来酰亚胺氮氧自由基对多种活细胞,如 CHO-I、CHO-II、肝癌细胞进行标记, 并且标记了食道和食道癌的组织块. 我们的实验表明, 所有被标记的活组织的波谱都呈现出 强固定化和弱固定化两种波谱叠加的五条谱线的 ESR 波谱. 马来酰亚胺自旋标记物能与硫 氢基作特异性的共价结合, 其 ESR 谱直接反应了硫氢基的物理状态.

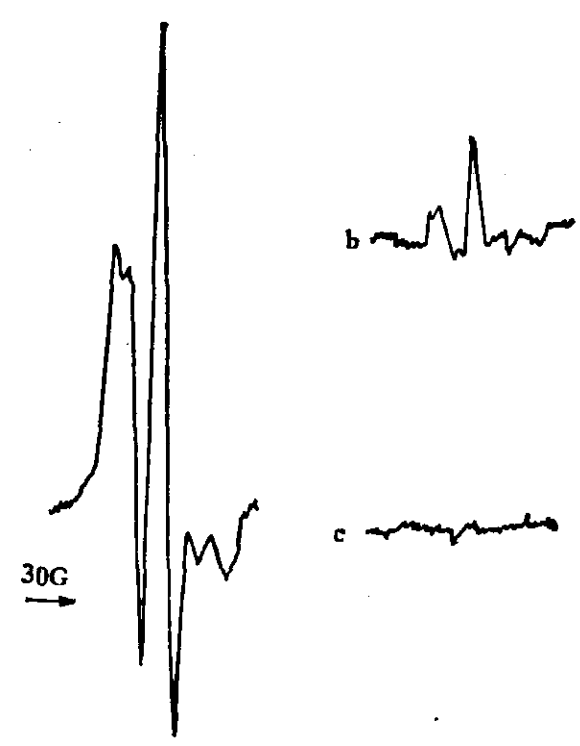

图 3 马来酰亚胺氮氧自由基标记羊红细胞膜的 ESR 波谱

a. 正常标记; b. $\mathrm{HgCl}_{2}$ 封闭; c. 未标记的膜

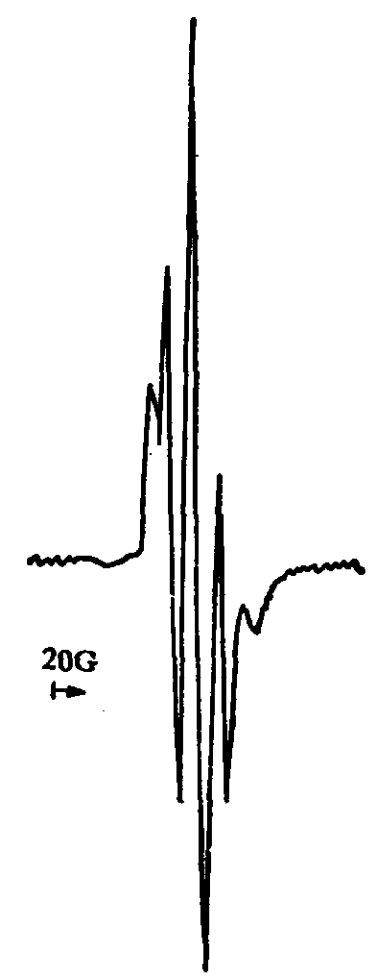

图 4 马来酰亚胺氮篻自由基标记 CHO-1 细胞 1 小时后的 ESR 波谱 


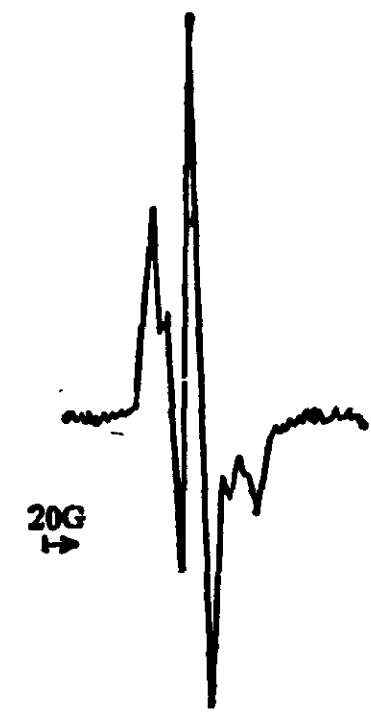

图 5 马来酰亚胺唤笋自由基 标记 CHO-I 细胞 16 小时后 的 ESR 波谱

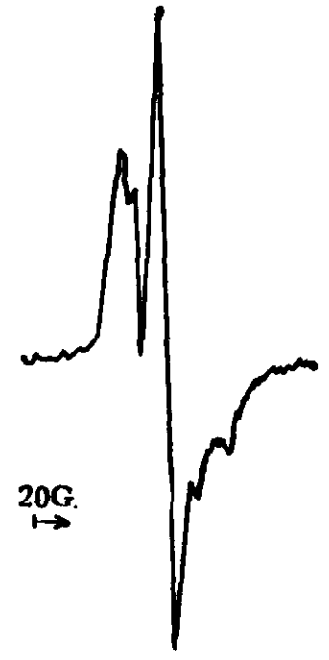

图 6 CHO-II 细胞经马来酰 亚胺氮续自由基标记 1 小时后 加铁氯化钾所得的 ESR 谱

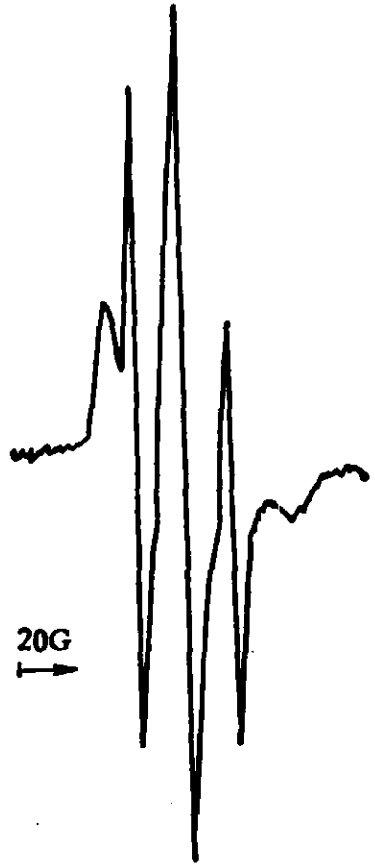

图 7 CHO-II 细胞经马来酰 亚胺氮等自由基标记 1 小时 后的 ESR 措

图 4 和图 5 分别为 CHO-I 细胞被自旋标记经 1 小时及 16 小时后测得的 ESR 谱. 标 记 16 小时后测定的 CHO-I 细胞的 ESR 波谱的强固定化组分的相对强度增高. 此实验证 明自旋标记物随着时间的推延逐步深入到活细胞内部结构,与处于深层的硫氢基相结合,其运 动受到限制,因此波谱的强固定化组分增强.

我们使用了谱线增宽剂 $\mathrm{K}_{3} \mathrm{Fe}(\mathrm{CN})_{6}{ }^{[8]}(0.25 \mathrm{M})$, 进一步证明了马来酰亚胺氮氧自由基 标记了处于两种不同环境的硫氢基团. 图 6 为 $\mathrm{CHO}-\mathrm{II}$ 细胞被自旋标记经 1 小时后加 $\mathrm{K}_{3} \mathrm{Fe}(\mathrm{CN})_{6}$ 测定的 ESR 波谱. 与图 7 相比,弱固定化成份减弱. 增宽剂的波谱效应是由于过 渡金属离子的存在，产生了物理的相互作用，从而导致谱线的增宽. 另外，因为 $\mathrm{K}_{3} \mathrm{Fe}(\mathrm{CN})_{6}$ 对 细胞的不渗透性,只能使细胞表面自旋标记物的 ESR 信号猝灭,所以致使弱固定化组分减弱. 于是更进一步证明马来酰亚胺氮氧自由基确实标记了处于膜的表层和内层的两种不同环境的 硫氢基团.

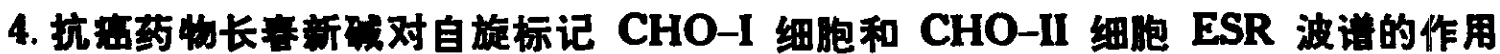

图 8 表明, 在经长春新碱预处理的与未经处理的自旋标记 CHO-I 型细胞的 ESR 波谱之间 无明显差别;相反地,在经长春新碱预处理的与未经处理的自旋标记 CHO-II 细胞的 ESR 波 谱之间有明显差异，这表现在弱固定化对强固定化组分的谱线高度的比值上．经计算得到以 下数值:

图 8 中 a. $\mathrm{A} / \mathrm{B}=9.65 ;$ b. $\mathrm{A} / \mathrm{B}=9.00$; c. $\mathrm{A} / \mathrm{B}=9.76 ; \mathrm{d} . \mathrm{A} / \mathrm{B}=4.33$.

其中 $\mathrm{A}$ 一一弱固定化组分低场线的高度，即波谱由低场第二个蜂的高度；B-一强固定 化组分低场线的高度，即波谱由低场第一个峰的高度。

由上面数值可以看出，经长春新碱预处理后的自旋标记 CHO-II 型细胞 ESR 波谱的弱 固定化对强固定化组分谱线高度的比值较对照样品明显下降. 而在同样经过长春新碱预处理 


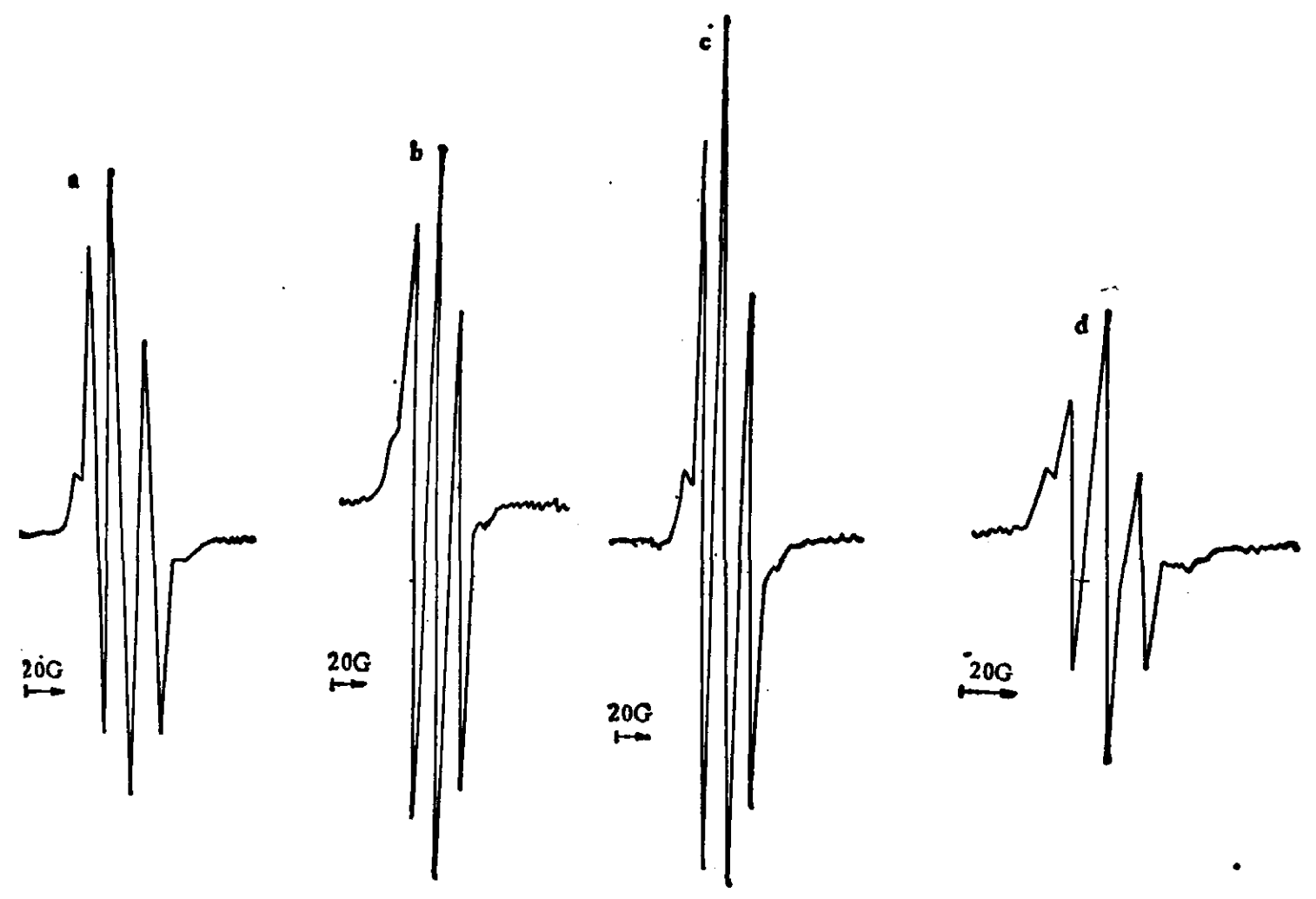

图 8 马来酰亚胺自旋标记 CHO-I 和 CHO-II 细胞的 ESR 波谱 a. CHO-I 细胞; b. 经长春新碱预处理的 CHO-I 细胞; c. CHO-II 细胞; d. 经长春新碱预处理的 CHO-II 细胞

的 CHO-I 型细胞及其对照之例中则无明显变化,我们重复了多次实验,均得相似的结果.

此实验证明, 转化细胞 CHO-II 型对抗癌药物长春新碱的敏感性明显地高于相对正常的 细胞 CHO-I 型. 而这种抗癌药物作用的分子机理显然与细胞中硫氢基团的变化有直接关系.

致谢：本文承贝时璋教授审阅，并提出宝贵意见．万谦、卢景芬、呼俊改同志提供 ESR 技术帮助, 江玲 同志供给培养细胞,特此表示衷心感谢。

\section{考文僺}

[1] Swartz, H. M., Bolton, J. R. \& Borg, D. C. ed., in Biological Applications of Electron Spin Resonance, Wiley-Interscience, New York, 1972, 483-539.

$\lceil 2\rceil$ Griffith, O. H. \& MeConnell, H. M., Proc. Natl. Acad. Sci. USA, 55(1966), 10.

[ 3 ] Sandberg, H. E., Bryant, R. G. \& Piette, L. H., Arch. Biochem. Biophys., 133(1969), 144.

[4] Lee, N., Scandella, C. \& Inouye, M., Proc. Natl. Acad. Sci. USA, 75(1978), 127.

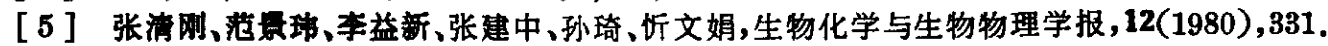

[ 6 ] Berliner, L. J. ed., Spin Labeling, Theory and Applications, Academic Press, New York, 1976. 5-58.

[ 7 ] Hsia, J. C. \& Piette, L. H., Arch. Biochem. Biophys., 129(1969), 296.

[ 8 ] Lepock, J. R., Morse II, P. D., Mehlhorn, R. J., Hammerstedt, ;R. H., Snipes, W. \& Keith, A. D., FEBB Lett., 60(1975), 185. 